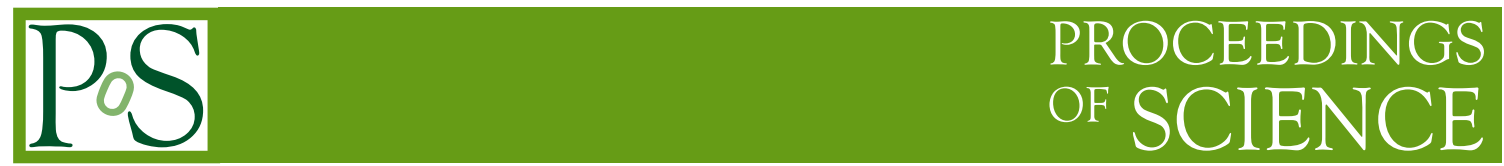

\title{
Thermal dilepton production from hot QCD
}

\author{
M. Laine ${ }^{* \dagger}$ \\ Institute for Theoretical Physics, AEC, University of Bern, Sidlerstrasse 5, 3012 Bern, \\ Switzerland \\ E-mail: laineditp.unibe.ch
}

NLO and LPM-resummed computations of thermal dilepton production from a hot QCD plasma are reviewed. An interpolation applicable for all positive photon invariant masses is presented, and possibilities for comparisons with lattice and experimental data are pointed out.

9th International Workshop on Critical Point and Onset of Deconfinement

17-21 November, 2014

ZiF (Center of Interdisciplinary Research), University of Bielefeld, Germany

\footnotetext{
* Speaker.

${ }^{\dagger}$ Supported in part by the Swiss National Science Foundation (SNF) under grant 200020-155935.
} 


\section{Introduction and observables}

The thermal production rate of off-shell photons, subsequently decaying into on-shell leptonantilepton pairs, is a rich source of information concerning the properties of the hot QCD medium generated in heavy ion collision experiments. If invariant masses corresponding to known vacuum resonances are avoided, the result can be expected to be relatively free from non-thermal background effects. Conversely, a focus on thermal modifications of prominent resonances, particularly quarkonium states, can in itself be turned into a useful probe of medium properties. In the present contribution we concentrate on non-resonant production and in particular on the contribution from gluons and three $\left(N_{\mathrm{f}}=3\right)$ deconfined light quark flavours.

In order to establish notation, let us denote by $T$ the temperature; by $k \equiv|\mathbf{k}|$ the total momentum of the dilepton pair with respect to the plasma rest frame; by $k_{0}$ the pair's energy; and by

$$
M \equiv \sqrt{k_{0}^{2}-k^{2}}
$$

its invariant mass. To leading order in the electromagnetic fine-structure constant $\alpha_{e}$ and for massless quarks, the differential production rate per volume reads $[1,2,3]$

$$
\frac{\mathrm{d} \Gamma_{\mu^{-} \mu^{+}}}{\mathrm{d} k_{0} \mathrm{~d}^{3} \mathbf{k}} \stackrel{M^{2}}{=} m_{Z}^{2}-\frac{n_{\mathrm{B}}\left(k_{0}\right)}{3 \pi^{3} M^{2}} \theta\left(M^{2}-4 m_{\mu}^{2}\right)\left(1+\frac{2 m_{\mu}^{2}}{M^{2}}\right)\left(1-\frac{4 m_{\mu}^{2}}{M^{2}}\right)^{\frac{1}{2}} \alpha_{e}^{2} \sum_{i=1}^{N_{\mathrm{f}}} Q_{i}^{2} \operatorname{Im} \Pi_{\mathrm{R}}\left(k_{0}, k\right) .
$$

Here $n_{\mathrm{B}}$ is the Bose distribution, $Q_{i}$ the electric charge of a quark of flavour $i$ in units of the electron charge, and $\operatorname{Im} \Pi_{R}$ stands for the imaginary part of a retarded correlator (i.e. a spectral function) of one flavour, evaluated in an equilibrium ensemble at a temperature $T$.

Given that we are interested in making contact with lattice simulations, the retarded correlator is best interpreted as an analytic continuation of an imaginary-time correlator (cf. e.g. ref. [4]). Denoting the imaginary-time coordinates by

$$
X \equiv(\tau, \mathbf{x}), \quad K \equiv\left(\omega_{n}, \mathbf{k}\right)
$$

with $\omega_{n} \equiv 2 \pi T n, n \in \mathbb{Z}$, the analytic continuation reads

$$
\Pi_{\mathrm{R}}\left(k_{0}, k\right) \equiv \int_{0}^{1 / T} \mathrm{~d} \tau e^{i \omega_{n} \tau} \int_{\mathbf{x}} e^{i \mathbf{k} \cdot \mathbf{x}}\left\langle J^{\mu}(X) J_{\mu}(0)\right\rangle_{\omega_{n} \rightarrow-i\left[k_{0}+i 0^{+}\right]}, \quad J^{\mu} \equiv \bar{\psi} \gamma^{\mu} \psi,
$$

where the spinor $\psi$ represents one flavour, and a suitable ultraviolet regularization is needed for defining the Fourier transform at short separations. It is interesting to also consider the space-like correlator

$$
V_{\mu v}\left(\omega_{n}, z\right) \equiv \int_{0}^{1 / T} \mathrm{~d} \tau e^{i \omega_{n} \tau} \int \mathrm{d}^{2} \mathbf{x}_{\perp}\left\langle J_{\mu}(X) J_{v}(0)\right\rangle, \quad \mathbf{x} \equiv\left(\mathbf{x}_{\perp}, z\right)
$$

Clearly,

$$
\Pi_{\mathrm{R}}\left(k_{0}, k\right)=\left\{\int_{-\infty}^{\infty} \mathrm{d} z e^{i k z} V_{\mu}^{\mu}\left(\omega_{n}, z\right)\right\}_{\omega_{n} \rightarrow-i\left[k_{0}+i 0^{+}\right]} .
$$

The last equation suggests that spatial correlations measured with $\omega_{n} \neq 0$ have a principal relation to the real-time dilepton production rate captured by $\operatorname{Im} \Pi_{R}$. 


\section{Different regimes and previous work}

If eq. (1.4) is addressed within the weak-coupling expansion, the method to be used depends on the parametric magnitudes of $k$ and $M$. In the following we recall the main cases, denoting by $g \equiv \sqrt{4 \pi \alpha_{\mathrm{s}}}$ the gauge coupling. For reference, the leading-order result reads

$$
-\operatorname{Im} \Pi_{\mathrm{R}}=\frac{N_{\mathrm{c}} T M^{2}}{2 \pi k} \ln \left\{\frac{\cosh \left(\frac{k_{+}}{2 T}\right)}{\cosh \left(\frac{k_{-}}{2 T}\right)}\right\}, \quad k_{ \pm} \equiv \frac{k_{0} \pm k}{2} .
$$

The simplest case to discuss is $k=0$, because then the result only depends on a single kinematic variable, $M=k_{0}$. If $M \gtrsim \pi T$, then the NLO correction to eq. (2.1) is infrared finite and small $[5,6,7]$ (these results have recently been extended to a finite quark mass [8]). However, the NLO correction increases rapidly as $M$ decreases; for $M \sim g T$, the correction is of $O(1)$ and needs to be summed to all orders, yielding a large enhancement [9]. More recently, it has been realized that the original ("HTL") resummation is not sufficient for capturing all relevant effects for $M \lesssim g T$. The correct infrared behaviour, including a transport peak in $\operatorname{Im} \Pi_{\mathrm{R}} / k_{0}$ whose width is $\sim g^{4} T / \pi^{3}$ and whose height determines the electric conductivity $\sim \alpha_{e} T / g^{4}$, has been worked out in numerical form in ref. [10].

The phenomenologically perhaps most interesting case concerns the production rate of dilepton pairs with a "soft" invariant mass $(M \sim g T)$ but large spatial momentum $(k \gtrsim \pi T)$. In this regime the NLO-rate has a logarithmic singularity, which is regulated (as indicated below by $M \rightarrow g T$ ) by Landau damping of the spacelike quarks mediating $t$-channel exchange [11, 12]:

$$
-\operatorname{Im} \Pi_{\mathrm{R}}=\ldots+\frac{\alpha_{\mathrm{S}} N_{\mathrm{c}} C_{\mathrm{F}} T^{2}}{2} \ln \left(\frac{T}{M \rightarrow g T}\right)\left[1-2 n_{\mathrm{F}}(k)\right],
$$

where $n_{\mathrm{F}}$ is a Fermi distribution. In addition, there are finite terms which all contribute at the same order because of collinear enhancement, and need to be handled through Landau-PomeranchukMigdal (LPM) resummation [13] (LPM resummation incorporates HTL resummation in an approximation valid for $k \gg g T$ ). In order to avoid double counting, LPM resummation needs to be carefully combined with other processes [14].

In a "hard" regime $M \gg \pi T$, Operator Product Expansion (OPE) techniques become applicable [15]. The result is available in a closed form up to NLO:

$$
-\operatorname{Im} \Pi_{\mathrm{R}}=\frac{N_{\mathrm{c}} M^{2}}{4 \pi}\left(1+\frac{3 \alpha_{\mathrm{s}} C_{\mathrm{F}}}{4 \pi}\right)+\frac{4 \alpha_{\mathrm{s}} N_{\mathrm{c}} C_{\mathrm{F}}}{9}\left(1+\frac{4 k^{2}}{3 M^{2}}\right) \frac{\pi^{2} T^{4}}{M^{2}}+\mathscr{O}\left(\frac{\alpha_{\mathrm{s}} T^{6}}{M^{4}}\right) .
$$

Yet another approach is to carry out lattice simulations. Lattice QCD being formulated in imaginary time, with a time coordinate $0<\tau<1 / T$, it is however not possible to measure the rate directly, but rather a particular transformation thereof:

$$
G_{\mathrm{E}}(\tau, k)=\int_{0}^{\infty} \frac{\mathrm{d} k_{0}}{\pi} \operatorname{Im} \Pi_{\mathrm{R}}\left(k_{0}, k\right) \frac{\cosh \left(\frac{1}{2 T}-\tau\right) k_{0}}{\sinh \left(\frac{k_{0}}{2 T}\right)} .
$$

This means that a part of the contribution comes from the "unphysical" domain $k_{0}<k$. Even though it is in principle possible to invert the relation in eq. (2.4) for $\operatorname{Im} \Pi_{\mathrm{R}}\left(k_{0}, k\right)$ [4], in practice large systematic uncertainties are induced [16]. A more controlled approach is to insert an analytically determined $\operatorname{Im} \Pi_{\mathrm{R}}\left(k_{0}, k\right)$ into eq. (2.4) and compare the resulting $G_{\mathrm{E}}(\tau, k)$ directly with numerical measurements (for recent work and references, see ref. [17]). 


\section{Methods for recent developments}

We now turn to works whose scope can be summarized as follows:

(i) The rate $\operatorname{Im} \Pi_{\mathrm{R}}$ has been determined up to NLO in a "generic" regime $k, M \sim \pi T$, verifying the cancellation of infrared divergences and finding in general a small correction $[18,19]$.

(ii) For a hard momentum $k \sim \pi T$ there is an additional parametric scale, $M \sim g^{1 / 2} T$, across which NLO results valid for $M \gtrsim \pi T$ and LPM-resummed results valid for $M \lesssim g T$ can be interpolated into each other [20].

(iii) It has been suggested that the relation in eq. (1.6) can be turned into a non-trivial crosscheck of dilepton production rate computations, in the sense that the screening masses associated with the $|z| \gg 1 / T$ behaviour $V_{\mu \nu}\left(\omega_{n}, z\right)$ can be measured non-perturbatively on one hand, and computed through an LPM-type resummation on the other. This permits for a direct comparison of the two approaches, without analytic continuation [21]. It has also been speculated, and demonstrated within the AdS/CFT framework, that a direct "analytic continuation" of screening masses (rather than correlation functions) might allow for a determination of the electric conductivity [22].

(iv) Finally, in an impressive computation, the LPM-resummed analysis of ref. [13] has been extended up to NLO in the soft regime $M \lesssim g T$, verifying the predictions' stability [23].

The methods used in these studies are as follows. For (i), standard (unresummed) perturbation theory is sufficient, with the only complication that because of infrared divergences appearing in "real" and "virtual" corrections, intermediate stages of the results need to be worked out in the presence of an infrared regulator, which cancels in the end [18, 19].

For (ii), an essential ingredient is to realize that the result of the soft regime consists of two parts, the LPM-resummed one and another part, referred to as $2 \rightarrow 2$ scatterings, which also appears in the non-resummed NLO expression. So, for a consistent result in the soft regime, the LPMresummed result and the NLO result need to be summed together, but only after subtracting those terms from the NLO result which got resummed into the LPM one. This can be summarized as

$$
\left.\left.\operatorname{Im} \Pi_{\mathrm{R}}\right|_{\text {interpolant }} \equiv \operatorname{Im} \Pi_{\mathrm{R}}\right|_{\mathrm{NLO}}-\left.\operatorname{Im} \Pi_{\mathrm{R}}\right|_{\mathrm{LPM}} ^{\text {expanded to NLO }}+\left.\operatorname{Im} \Pi_{\mathrm{R}}\right|_{\mathrm{LPM}} ^{\text {full }} .
$$

This expression is correct in the soft regime $M \lesssim g T$ because the subtraction removes the danger of double-counting terms appearing in $\left.\operatorname{Im} \Pi_{\mathrm{R}}\right|_{\mathrm{NLO}}$, but also in the hard regime $M \gtrsim \pi T$, because the two variants of the LPM expression cancel against each other there (up to higher-order corrections).

For (iii), two ingredients are needed. One is a theoretical analysis showing that a perturbative determination of the screening masses at $\omega_{n} \neq 0$ involves solving inhomogeneous Schrödingertype equations which are just analytic continuations of the corresponding equations appearing in LPM resummation (with, in particular, the same "potential" [24, 25] and the same "inhomogeneous terms"). The other ingredient is measuring screening masses with standard lattice QCD; in ref. [21] this was done for $N_{\mathrm{f}}=2$ light dynamical flavours.

For (iv), the parameters appearing in LPM resummation, in particular the asymptotic thermal masses and the potential, need to be modified through NLO corrections. Many ingredients are known from previous work [24, 26, 27], but need to be put together in a consistent fashion [23]. 




Figure 1: A comparison of screening masses related to different polarization states of the vector current in the presence of a Matsubara frequency $\left(\omega_{n}=2 \pi T n\right)$ in dynamical $N_{\mathrm{f}}=2 \mathrm{QCD}$ at $T \sim 250 \mathrm{MeV}$ (from ref. [21]). The correlation functions were measured in the direction of the $z$-axis, and were averaged over the "transverse" $\left(x_{1}, x_{2}\right)$ plane. The good agreement between lattice measurements (" "at") and resummed perturbative results (" pert") suggests that the systematic uncertainties of both approaches are moderate.

\section{Results}

In fig. 1, screening masses measured in $N_{\mathrm{f}}=2$ lattice QCD at $T \approx 250 \mathrm{MeV}$ are compared with corresponding predictions from perturbation theory, based on solving an LPM-type Schrödinger equation. It is seen that even at this "low" temperature, reachable in the current generation of LHC experiments, resummed perturbation theory does reproduce the qualitative features of the lattice measurements, with differences only on the $\sim 15 \%$ level. (It would be interesting to increase the resolution of the simulations, to take the continuum limit, and to study several temperatures, in order to see if the remaining discrepancies decrease.)

In fig. 2 a result for $\operatorname{Im} \Pi_{\mathrm{R}}$ based on eq. (3.1) is plotted as a function of $M$ and $k$. A crossover from one type of behaviour at $M \lesssim T$ to another shape at $M \gtrsim \pi T$ is clearly visible; as discussed above, the crossover takes parametrically place at $M \sim g^{1 / 2} T$.

Finally, in fig. 3 the physical dilepton rate from eqs. (3.1), (1.2) is illustrated. The grey bands indicated the uncertainty associated with variations of the renormalization scale. Even though the uncertainty is $\gtrsim 50 \%$ for $M \lesssim 1 \mathrm{GeV}$, the overall shape of the curves as well as their general normalization can be predicted. We also note that a recent study of the soft regime up to NLO [23] increases the results by $\mathscr{O}(10 \%$ ) for general parameters (up to $100 \%$ for $M=0.25 \mathrm{GeV}$ ), which suggests that the grey bands do indeed capture the magnitude of uncertainties.

\section{Outlook}

There are two major "applications" for the results reviewed here. First of all, curves such 


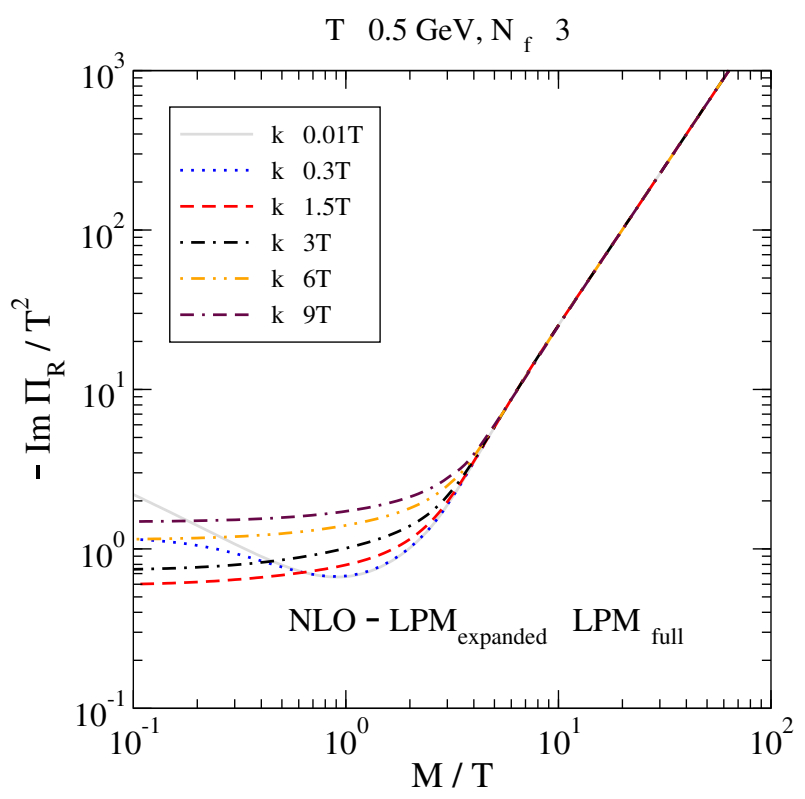

Figure 2: The vector channel spectral function determined through eq. (3.1) (from ref. [20]).

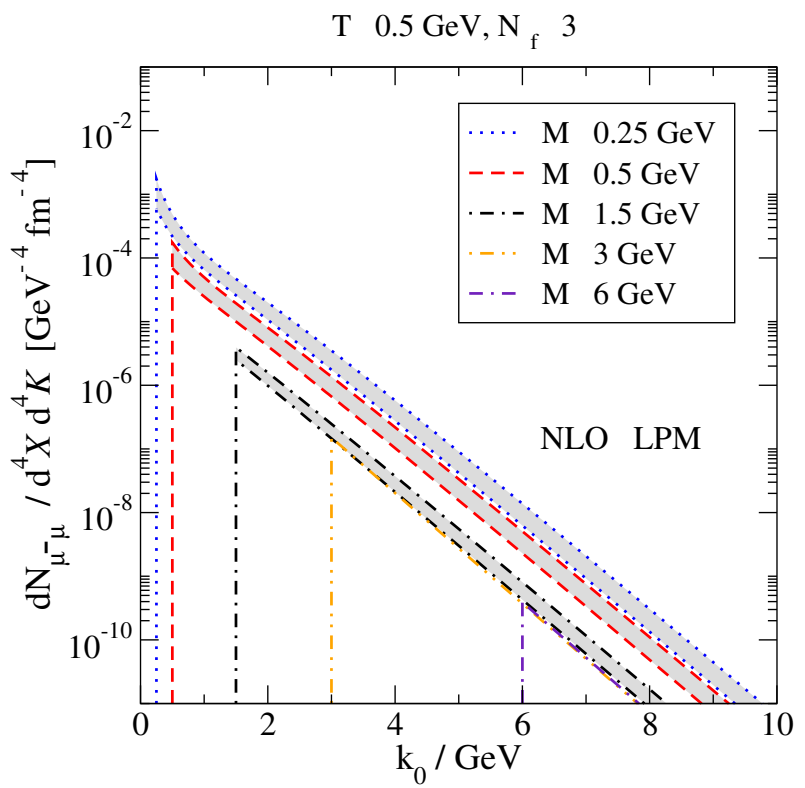

Figure 3: Physical $\mu^{-} \mu^{+}$production rates based on eqs. (3.1), (1.2) (from ref. [20]).

as shown in fig. 2, complemented by results for the regime $k_{0}<k$, can be inserted into eq. (2.4) and compared with direct lattice measurements. Compilations suitable for this purpose, based on refs. [7, 10, 16, 19, 20, 23], can be downloaded from ref. [28]. Second, results such as shown in fig. 3 could be inserted into hydrodynamical models such as ref. [29], and compared with experimental data from heavy ion collision experiments. Data suitable for this purpose, from refs. [20, 23], can be downloaded from ref. [30]. 


\section{References}

[1] L.D. McLerran and T. Toimela, Phys. Rev. D 31 (1985) 545.

[2] H.A. Weldon, Phys. Rev. D 42 (1990) 2384.

[3] C. Gale and J.I. Kapusta, Nucl. Phys. B 357 (1991) 65.

[4] G. Cuniberti et al, Commun. Math. Phys. 216 (2001) 59 [cond-mat/0109175].

[5] R. Baier, B. Pire and D. Schiff, Phys. Rev. D 38 (1988) 2814.

[6] Y. Gabellini, T. Grandou and D. Poizat, Annals Phys. 202 (1990) 436.

[7] T. Altherr and P. Aurenche, Z. Phys. C 45 (1989) 99.

[8] Y. Burnier, 1410.1304.

[9] E. Braaten, R.D. Pisarski and T.-C. Yuan, Phys. Rev. Lett. 64 (1990) 2242.

[10] G.D. Moore and J.-M. Robert, hep-ph/0607172.

[11] J.I. Kapusta et al, Phys. Rev. D 44 (1991) 2774 [Erratum-ibid. D 47 (1993) 4171].

[12] R. Baier, H. Nakkagawa, A. Niégawa and K. Redlich, Z. Phys. C 53 (1992) 433.

[13] P. Aurenche, F. Gelis, G.D. Moore and H. Zaraket, JHEP 12 (2002) 006 [hep-ph/0211036].

[14] M.E. Carrington, A. Gynther and P. Aurenche, Phys. Rev. D 77 (2008) 045035 [0711.3943].

[15] S. Caron-Huot, Phys. Rev. D 79 (2009) 125009 [0903.3958].

[16] Y. Burnier and M. Laine, Eur. Phys. J. C 72 (2012) 1902 [1201.1994].

[17] H.T. Ding, O. Kaczmarek and F. Meyer, 1412.5869.

[18] M. Laine, JHEP 05 (2013) 083 [1304.0202].

[19] M. Laine, JHEP 11 (2013) 120 [1310.0164].

[20] I. Ghisoiu and M. Laine, JHEP 10 (2014) 83 [1407.7955].

[21] B.B. Brandt, A. Francis, M. Laine and H.B. Meyer, JHEP 05 (2014) 117 [1404.2404].

[22] B.B. Brandt, A. Francis, M. Laine and H.B. Meyer, Nucl. Phys. A 931 (2014) 861 [1408.5917].

[23] J. Ghiglieri and G.D. Moore, JHEP 12 (2014) 029 [1410.4203].

[24] S. Caron-Huot, Phys. Rev. D 79 (2009) 065039 [0811.1603].

[25] M. Panero, K. Rummukainen and A. Schäfer, Phys. Rev. Lett. 112 (2014) 162001 [1307.5850].

[26] S. Caron-Huot, Phys. Rev. D 79 (2009) 125002 [0808.0155].

[27] J. Ghiglieri et al, JHEP 05 (2013) 010 [1302.5970].

[28] J. Ghiglieri and M. Laine, web page Data for the thermal vector channel spectral function, http://www.laine.itp.unibe.ch/dilepton-lattice/.

[29] G. Vujanovic et al, Phys. Rev. C 89 (2014) 034904 [1312.0676].

[30] M. Laine, web page Data for the interpolated dilepton rate, http: //www.laine.itp.unibe.ch/dilepton-lpm/. 